\title{
Pediatric Mortality Due to Nosocomial Infection: A Critical Approach
}

Julia Marcia Maluf Lopes ${ }^{1,2,3}$, Eugenio Marcos Andrade Goulart ${ }^{1}$ and Carlos Ernesto Ferreira Starling ${ }^{3}$ ${ }^{1}$ Department of Pediatrics, School of Medicine of the Federal University of Minas Gerais; ${ }^{2}$ General Center of Pediatrics (Centro Geral de Pediatria - CGP) of Hospital Foundation of Minas Gerais (FHEMIG); ${ }^{3}$ Nosocomial Infection Control Advisory Board of FHEMIG; Belo Horizonte, MG, Brazil

\begin{abstract}
Nosocomial infection is a frequent event with potentially lethal consequences. We reviewed the literature on the predictive factors for mortality related to nosocomial infection in pediatric medicine. Electronic searches in English, Spanish and Portuguese of the PubMed/MEDLINE, LILACS and Cochrane Collaboration Databases was performed, focusing on studies that had been published from 1996 to 2006. The key words were: nosocomial infection and mortality and pediatrics/neonate/newborn/child/infant/adolescent. The risk factors found to be associated with mortality were: nosocomial infection itself, leukemia, lymphopenia, neutropenia, corticosteroid therapy, multiple organ failure, previous antimicrobial therapy, catheter use duration, candidemia, cancer, bacteremia, age over 60, invasive procedures, mechanical ventilation, transport out of the pediatric intensive care unit, methicillin-resistant Staphylococcus aureus, Pseudomonas aeruginosa, and Burkholderia cepacia infections, acute physiology and chronic health evaluation (APACHE) II scores over 15. Among these factors, the only one that can be minimized is inadequate antimicrobial treatment, which has proven to be an important contributor to hospital mortality in critically-ill patients. There is room for further prognosis research on this matter to determine local differences. Such research requires appropriate epidemiological design and statistical analysis so that pediatric death due to nosocomial infection can be reduced and health care quality improved in pediatric hospitals.
\end{abstract}

Key-Words: Nosocomial infection, mortality, prognosis, healthcare quality, pediatric hospital.

Nosocomial infection (NI) is an important public-health problem in Brazil; considering 11 million hospital admissions per year and a $5 \%$ to $10 \%$ NI rate, we project 550,000 to $1,100,000$ cases, with all their associated costs, including financial, patient suffering and mortality [1]. The current world-wide focus on improving the cost-effectiveness of health care, along with reports of successful nosocomialinfection-control programs stimulates hospital administrators, infection-control teams and researchers to try to understand their own local situations in order to improve health-care programs. Knowledge about the prognosis of mortality related to NI would allow prior interventions and more targeted and effective infectioncontrol programs. Haley and his colleagues indicated that NI are among the top-ten-leading causes of death in the United States [2].

From a global perspective, acknowledgment that NIs do occur and that many of these infections are preventable is an obvious prerequisite for improvements in infection control in any country. Infection-control professionals require training and experience in a complex amalgam of infectious diseases, epidemiology, microbiology, biostatistics, informatics, healthcare management, patient-care practices, adult education and behavioral science [3].

Health-care workers have always been concerned about $\mathrm{NI}$, and so have regulatory agencies and organizations. It

Received on 25 February 2007; revised 30 August 2007.

Address for correspondence: Dr. Julia Marcia Maluf Lopes Rua Contria. 1500, ap. 404 A. Zip code: 30.430-460 Belo Horizonte, MG, Brazil. Phone/Fax: 55-31-3239-9045; Phone: 55-31-3372-6021; 3239- 9008 E-mail: JuliaLopes@vsnet.com.br.

The Brazilian Journal of Infectious Diseases 2007;11(5):515-519. (C) 2007 by The Brazilian Journal of Infectious Diseases and Contexto Publishing. All rights reserved. never will be reduced to zero, but many of its causes are related to a lack of applying adequate methodology to control it. Nosocomial infection and associated mortality rates are important health-care quality indicators. Evidence-based health care is the conscientious, explicit, and judicious use of current best evidence from healthcare research in making decisions that fit the circumstances and wishes of individual patients or groups [4].

Brazilian federal regulations published in 1992 and 1998 made it obligatory to report lethality and mortality rates in NIcontrol programs; however, this was done without stipulating a uniform and viable methodology $[5,6]$. The National Nosocomial Infection Surveillance (NNIS) System requires hospitals to provide high-quality data on NI [7]. This government agency has its own specific definitions and also applies Centers for Disease Control and Prevention (CDCAtlanta) definitions [8,9].

In order, to develop adequate legislation and to support continuous healthcare-quality improvement programs, it is crucial to obtain data and indicators on NI and associated mortality by means of methodologically well-designed research that will be accepted by the scientific community. We reviewed the literature on the predictive factors for mortality related to NI in Pediatrics, focusing on studies that were published from 1996 to 2006.

\section{Search Strategy}

Electronic search in English, Spanish and Portuguese of the PubMed/ MEDLINE, LILACS and Cochrane Collaboration Databases was performed, focusing on studies that had been published from 1996 to 2006 . The key words were: nosocomial infection and mortality and pediatrics/neonate/newborn/child/ infant, adolescent. 


\section{The Studies}

A six-year prospective study of children involved in 374 hospital and community-acquired episodes of Gram-negative bacteremia in a tertiary-care children's medical center in Israel reported a crude mortality of $11.4 \%$. The increased mortality was significantly associated with acute leukemia, neutropenia, hospital-acquired infections and previous corticosteroid therapy ( $p=0.03,0.003,0.006$ and 0.01 , respectively) and inadequate antimicrobial treatment and shock $(p=0.001)$. Statistical analysis was performed applying Chi-square and Wilcoxon's tests [10]. A descriptive follow-up study of adult and pediatric patients (204,598 discharges), made from 1991 to 1995 , found a mortality rate related to NI of $7.5 \%$ and an overall mortality rate of $0.3 \%$ [1].

During a 22-month period, a prospective multicenter observational study in a cohort of 145 patients with candidemia (defined as the growth of Candida species from at least one blood culture taken from a peripheral vein) found the following risk factors for death, based on univariate analysis: age over 64, catheter retention, candidemia due to species other than Candida parapsilosis, hypotension, poor performance status and no antifungal treatment. Based on logistic regression multivariate analysis, older age (odds ratio [OR ] 1.02; $\mathrm{p}=0.02$ ), and non-removal of a central venous catheter (OR: 4.81; $\mathrm{p}<$ 0.0001 ) were the only factors associated with an increased risk for death [11].

Based on a one-year prospective analysis, data from all patients with hospital-acquired Staphylococcus aureus bacteremia (SAB) admitted to four hospitals in Copenhagen County, Denmark, from May 1, 1994, through April 30, 1995, were evaluated. The presence of a central-venous catheter (OR=6.9; 95\% confidence interval [CI]:2.8-17.0), anemia (OR:3.3; 95\%CI:1.4-7.6), and hyponatremia(OR:3.3; 95\%CI:1.57.0) was significantly associated with hospital-acquired SAB in a conditional and in a normal logistic regression analysis. Nasal carriage was not an independent risk factor; however, nasal carriers among patients in surgery (OR:4.0; 95\%CI:1.313.0) had a significantly higher risk for hospital-acquired $S A B$ compared with matched and unmatched controls. The presence of hospital-acquired SAB increased the mortality rate 2.4-fold (95\%CI:1.1-5.2). The Cox regression model was applied, and the results demonstrated that hospital-acquired SAB in itself and age ( $>60$ years) increase mortality independently. The mortality-rate ratio for cancer was 1.7 (95\%CI:0.8-3.5). However, this was not significantly $(\mathrm{p}=0.14)$ different from baseline mortality [12].

A European, multicenter six-month prospective study, using CDC criteria, aimed to determine the incidence of NI in the various units by site of infection and bacterial epidemiology, found that mortality due to NI was 10\% (0 to $27.3 \%$ ) in pediatric-intensive-care units (PICU), 17\% in neonatal units and $1.5 \%$ for immunocompromised children. Variables were evaluated using the chi-square or Fisher's Exact Test for categorical variables and the Mann-Whitney test for continuous ones. They found large differences in NI frequency and microbial epidemiology and concluded that clinical monitoring of NIs and bacterial resistance profiles are necessary in all pediatric units [13].

A population-based case-control study of 277 postneonatal infant deaths caused by diarrhea and pneumonia examined how risk factors are related to the quality of medical assistance. Multiple-logistic-regression analysis showed the following factors to be independently associated with increased risk of postneonatal death: delayed immunization (OR:2.48; 95\%CI:1.17-5.23), critically ill at hospital admission (OR:10.94; 95\%CI:4.91-24.34), unperformed hospital procedures (OR:10.08; 95\%CI:3.55-20.59) and malnutrition at hospital admission (OR:3.58; 95\%CI:1.42-9.07). The authors concluded that low quality of medical assistance is an important risk factor among avoidable causes of post-neonatal deaths [14].

A prospective-cohort study was performed at St. Louis Children's Hospital, a 235-bed academic tertiary care center with a combined 22-bed medical and surgical PICU; the subjects were all the patients admitted to the PICU between September 1, 1999, and May 31, 2000, when a high rate of bloodstream infection was identified (BSI $=13.8$ per 1,000 central venous catheter days). In multiple logistic regression analysis, patients with BSI were more likely to have multiple central-venous catheters (adjusted odds ratio [aOR]:5.7; 95\%CI:2.9-10.9), arterial catheters (aOR:5.5; 95\%CI:1.8-16.3), invasive procedures performed in the PICU (aOR:4.0; 95\%CI:2.0-7.8), and were transported out of the PICU (aOR:3.4; 95\%CI:1.8-6.7) to the radiology or operating room suites. When severity of illness was measured at admission, giving a Pediatric Risk of Mortality (PRISM) score, underlying illnesses, and medications were not associated with increased risk of nosocomial BSI. The authors concluded that the risk factors were related more to the healthcare process than to severity of the illness and that additional research is needed to develop interventions to reduce nosocomial BSI in children [15].

A Brazilian study that reviewed epidemiological data from patients admitted to a PICU of a university hospital, with data collected, retrospectively, from all patients admitted between 1978 and 1994, showed a general mortality rate of 7.4\%. Data were presented as percentages and compared using a Chisquare test. The authors concluded that mortality was higher in malnourished children (relative risk [RR]:2.98; 95\%CI:2.643.36; $\mathrm{p}<0.0001$ ), in those younger than 12 months (RR:1.86; 95\%CI:1.65-2.10; $\mathrm{p}<0.0001)$ and that sepsis was the most main cause of death [16].

Another Brazilian descriptive study reviewed data from 69 deaths that occurred within 48 hours after admission in 1993 to examine a possible association between NI and mortality. NI was considered to be a secondary cause in 51\% of the deaths and a primary cause in 30\%, mostly among those not critically ill on hospital admission [17].

In a study of nosocomial $S$. aureus bacteremia in patients over 14 years of age, the authors developed a predictive model of mortality, comparing the beginning (39\%) and middle (33\%) 
1990s. They found no significant differences in the mortality rates. Based on logistic regression, septic shock, source of bacteremia and methicillin resistance were found to be positively associated with subsequent mortality [18].

A study to evaluate the predictive power of the PRISM score for development of NI among 341 patients admitted from June 1998 to December 2000 in a Brazilian PICU using stepwise logistic regression analysis found that the best predictors for NI were device utilization ratio (OR:4.64; 95\%CI:2.7-7.9; $\mathrm{p}<0.001)$ and prior antimicrobial therapy (OR:1.26; 95\%CI: 1.2-1.4; $\mathrm{p}<0.001$ ), and that PRISM score was not useful for predicting NI [19]. A paper from the Department of Critical Care Medicine, University of Pittsburgh School of Medicine reported that prolonged lymphopenia (absolute lymphocyte count $<1,000$ for $>7$ days) was associated independently with NI (OR: 5.5, 95\%CI: 1.7-17, p < 0.05), death (OR:6.8, 95\%CI:1.3-34, p < 0.05), and splenic and lymph node hypocellularity (OR: 42, 95\%CI:3.7-473, p < 0.05) [20].

\section{Limitations of the Various Studies to Address the Question}

We found no studies that focused only on pediatric patients, and methodologies varied, making comparisons difficult. Most studies were descriptive for rates or focused on neonatal population outbreaks, which was not our target. In some of them, multivariate analyses were performed using logistic regression, but these were used to find risk factors for NI, and not survival analyses of prognostic factors or attributable mortality.

Some studies examined NI related to certain infection sites or etiological agents [11]. Others focused on PICU or resistantbacteria infections. The first U.S. national multicenter description of antimicrobial use in neonatal intensive care units (NICUs) and PICUs reported a high prevalence of antimicrobial use among these patients; the authors concluded that assessment strategies targeting antimicrobial use in pediatrics are needed [21].

Characteristics of 42 episodes of B. cepacia bacteremia in 40 patients admitted to the Taipei Veterans General Hospital between January 1997 and December 1999 were retrospectively analyzed. Most of the patients had serious underlying diseases, such as diabetes mellitus, malignancy, congestive heart failure, and chronic obstructive pulmonary disease. The overall mortality rate was $29 \%$ $(12 / 42)$, and $44 \%(12 / 27)$ of all deaths were directly related to $B$. cepacia bacteremia [22].

Most of the studies took place in hospital units, where the population includes children of various ages as well as adults, with great heterogeneity of underlying diseases, from neonates with or without congenital abnormalities, to adolescents with or without traumas and immunocompromised patients. Therefore, patients who have been admitted to the same ward or PICU/ NICU may have different risk factors, because of their intrinsic-risk factors. This was quite clear, for instance, in a University of Pittsburgh School of Medicine study; prolonged lymphopenia and apoptosis-associated depletion of lymphoid organs played a role in nosocomial-sepsis-related death in critically-ill children [20].

In order to compare the different risk factors, there have been some attempts to standardize risk scores for mortality; but there is no consensus about the best means to do so. According to a cohort study undertaken at the Pontificia Universidade Católica do Rio Grande do Sul, Hospital São Lucas PICU, the Pediatric Risk of Mortality (PRISM) and the Pediatric Index of Mortality (PIM) scores both offer a good capacity for discriminating between survivors and moribund patients [23].

Taking into consideration that PICUs vary in admission criteria, in another study, the authors concluded that PRISM score was not useful for predicting NI and that preventive measures should focus, whenever possible, on reduction of invasive devices and of antimicrobial therapy [19]. Risk factors for BSI are more frequently associated with the healthcare process than with the severity of illness among PICU patients [15].

Data from a nationwide US, concurrent surveillance study (Surveillance and Control of Pathogens of Epidemiological Importance [SCOPE]), one of the largest multicenter studies performed to date, were used to examine the secular trends in the epidemiology and microbiology of nosocomial BSIs. The study detected 24,179 cases of nosocomial BSI in 49 hospitals over a seven-year period from March 1995 through September 2002 (60 cases per 10,000 hospital admissions). The crude mortality rate was $27 \%$. The authors concluded that the proportion of nosocomial BSIs due to antibiotic-resistant organisms is increasing in US hospitals [24].

The same trend may occur in Brazil, but we found no Brazilian data. Another North-American study to evaluate the relationship between inadequate antimicrobial treatment of infections (both community-acquired and nosocomial infections) and hospital mortality for patients requiring ICU admission found that the infection-related mortality rate for infected patients receiving inadequate antimicrobial treatment (42\%) was significantly greater than the infection-related mortality rate $(17.7 \%)$ of infected patients receiving adequate antimicrobial treatment (RR:2,37; 95\%CI: 1.83-3.08, p < 0.001). Using a logistic regression model, inadequate antimicrobial treatment of infection was found to be the most important independent determinant of hospital mortality for the entire patient cohort (aOR:4.27; 95\%CI:3.35-5.44, p <0.001). The other independent determinants of hospital mortality included the number of acquired organ-system derangements, use of vasopressor agents, the presence of an underlying malignancy, increasing APACHE II scores, increasing age, and a nonsurgical diagnosis at the time of ICU admission [25]. Another set of data suggested that administration of inadequate antimicrobial treatment to critically-ill patients with BSI is associated with a greater hospital mortality compared with adequate antimicrobial treatment of BSI and that clinical efforts should be aimed at reducing the administration of inadequate antimicrobial treatment to hospitalized patients 
with BSI, especially individuals infected with antibioticresistant bacteria and Candida species [26].

An Italian prospective-randomized-controlled trial conducted in a 20-bed tertiary referral NICU compared the effectiveness of a single dose and a three-day course of antibiotic prophylaxis in preventing bacterial infections in highrisk neonates. No significant differences were found between the two groups of neonates in mean birth weight, gestational age or postnatal age on admission. The incidence of vertical infection was similar in the two groups (16/67, 24\% vs. 14/63, $22 \%)$. Among the 130 newborns, 29 (22\%) acquired at least one nosocomial infection during their NICU stay; total hospital-acquired infections, calculated as the incidence density of infection (the number of infective episodes divided by the number of days in the NICU), were less frequent among newborns who received the three-day course than a single bolus (risk ratio [RR]:0.69). This difference, although not significant, was affected by the incidence density of confirmed nosocomial infections rather than by suspected infections (RR:0.59; 95\%CI 0.32-1.09; $\mathrm{p}=0.1$ ). There were no significant differences between the two groups in overall mortality. A single bolus administration on admission is therefore likely to be as effective as a three-day course of antibiotic prophylaxis in preventing bacterial infection in high-risk infants admitted to an NICU [27].

A retrospective study of 56 neonates admitted to a neonatal intensive care unit from 1996 to 2000 who had one or more blood cultures positive for Candida spp. examined the duration of candidemia in newborn infants and the incidence of persistent hospital-acquired candidemia and its associated morbidity and mortality compared with non-persistent candidemia. No significant differences were found between the infants with persistent and non-persistent candidemia in background or predisposing clinical factors. The authors concluded that persistent-neonatal candidemia is not associated with increased mortality [28].

An 11-year longitudinal prospective surveillance study in Australia and New Zealand to determine the incidence and mortality due to late-onset Gram-negative bacillary infections in neonatal units found that 702 of 3,113 (22.5\%) episodes of late onset sepsis in 681 infants were due to Gram-negative bacilli. Overall mortality was 21\% (142 of 681 infants), and it was significantly related to maturity, birth weight and infecting organism. Mortality was $25 \%$ for infants less than 30 weeks old compared with $11.5 \%$ for infants more than or equal to 30 weeks ( $\mathrm{p}<0.0001$ ) and $24 \%$ for infants with birth weights less than $1500 \mathrm{~g}$, versus $13 \%$ if more than or equal $1500 \mathrm{~g}(\mathrm{p}<$ 0.0001 ). Infection by $P$. aeruginosa was associated with $52 \%$ mortality (46 of 88 infants), significantly higher than the 14\% to $24 \%$ fatality from other Gram-negative bacilli $(\mathrm{p}<0.0001)$. During surveillance, the late onset Gram-negative bacillary infection rate remained stable at 1.14 per 1,000 live births (range $0.87-1.5)$. Similarly, mortality was unchanged, being 0.25 per 1000 live births (range 0.12-0.43). The authors concluded that Gram-negative bacilli are important causes of late onset neonatal sepsis, especially among very-low-birth-weight infants and provoke high mortality, particularly Pseudomonas aeruginosa infections [29].

In a prospective study at a Turkish university hospital, 93 ICU-acquired infections in 131 ICU patients were evaluated. Infection rates were found to be 70.9 per 100 patients and 56.2 per 1,000 patient-days. Pneumonia (35.4\%) and BSI (18.2\%) were the most common infections; S. aureus (30.9\%) and Acinetobacter spp. (26.8\%) were the most frequently-isolated microorganisms. The results of multivariate logistic regression analyses estimating the risk factors for ICU-acquired infections were as follows: length of stay in ICU ( $>7$ days) (OR:7.02; 95\%CI: 2.80-17.56), respiratory failure as a primary cause of admission (OR:3.7; 95\%Cl:1.41-9.70), sedative medication (OR:3.34; 95\%CI:1.27-8.79) and surgery (before or after admission to ICU; OR: 2.56; 95\% CI: 1.06-6.18). In logistic regression analyses, age (>60 years; OR:3.65; 95\%CI:1.48-9.0), APACHE II score >15(OR:4.67; 95\%CI:1.92-11.31), intubation (OR:3.60; 95\%CI:1.05-12.39) and central venous catheterization (OR:7.85; 95\%CI:1.61-38.32) were found to be significant risk factors for mortality. The difference in mortality rates between patients with ICU-acquired infection and uninfected patients was not significant (mortality rates: 42.3 and 45.6\%, respectively). A high incidence of nosocomial infections was found, and the risk factors for ICU-acquired infections and mortality were determined [30].

Brazilian reports are rare, especially those related to antimicrobial susceptibility patterns other than local data. Each hospital knows the most frequent local agents. Pseudomonas and Acinetobacter have had increasing resistance patterns. Pathogen frequency and resistance patterns (including Brazilian hospitals) have been studied, and a summary of results has been published [31]; nevertheless, there is a lack of recent data.

The first newsletter from ANVISA (the Brazilian health and drug monitoring agency) reported on a national pattern of NIs and on etiological agents and resistance patterns; though interpretation was difficult as various participating laboratories had no quality control. ANVISA emphasizes the need to use adequate methodology and development of procedures in each hospital [32].

These findings and the great variation in the ability of Latin-American laboratories to detect antimicrobial resistance patterns are the reason why international data are predominant even in Brazilian publications. A global Brazilian perspective remains a great challenge.

\section{Conclusions}

The articles that we reviewed showed that there have been advances in the efforts to prevent and control NIs. The number of reports in the international literature has increased in the last 10 years. The epidemiological design and the statistical analysis also have had a quality improvement throughout these years, going from descriptive to analytical studies. Nevertheless, some contradictory data and lacunae were 
found, especially in pediatrics and mortality related to or associated with NI. The risk factors varied from intrinsic to extrinsic, depending on where the research had taken place; they included the nosocomial infection itself, leukemia, lymphopenia, neutropenia, corticosteroid therapy, multipleorgan failure, previous antimicrobial therapy, catheter placement duration, candidemia, cancer, bacteremia, age over 60 , invasive procedures, mechanical ventilation, transport out of the pediatric-intensive-care unit, methicillin-resistant $S$. aureus, Gram-negative bacilli, P. aeruginosa, B. cepacia infections, acute physiology and chronic health evaluation (APACHE) II scores over 15.

Most important, emphasis was given to avoiding inadequate antimicrobial treatment of critically-ill patients. Among all the risk factors, the only one that normally can be manipulated is inadequate antimicrobial treatment, which has proven to be an important determinant of hospital mortality in critically-ill patients. Consequently, it is crucial to identify the etiological infectious agent quickly for rapid tailoring of antimicrobial treatment on the basis of culture results and the clinical course of the patient. There is still room for further prognosis research on this matter, examining local and national scenarios, in order to obtain a better understanding of the problem of pediatric mortality related to nosocomial infection.

\section{References}

1. Starling C.E.F., Couto B.R.G.M., Pinheiro S.M.C. Applying the Centers for Disease Control and Prevention and National Nosocomial Surveillance System methods in Brazilian hospitals. Am J Infect Control 1997;25:303-11.

2. Haley R.W., Quade D., Culver D.H. et al. The efficacy of infection surveillance and control programs in preventing nosocomial infections in US hospitals. Am J Epidemiol 1985;121:182-205.

3. Huskins W.C., Soule B.M. A global perspective on the past, present and future of nosocomial infection prevention and control. Am J Infect Control 1997;25:289-93.

4. Brouwers M.C., Haynes R.B., Jadad A.R., et al. Evidence-based health care and the Cochrane collaboration. Clinical Performance and Quality Health Care 1997;5:195-201.

5. Brazil. Ministry of Health. Regulation n. 930, aug 27,1992. Diário Oficial. Brasília, sept., 4. 1992.

6. Brazil Ministry of Health Regulation n. 2.616, may 12,1998. Diário Oficial, Brasília, may, 13. 1998.

7. Emori G.T., Culver D.H., Horan T.C., et al. National nosocomial infections surveillance system (NNIS): description of surveillance methodology. Am J Infect Control 1991;19:19-35.

8. Garner J.S., Jarvis W.R., Emori T.J., et al. CDC definitions for nosocomial infections. Am J Infect Control 1988;16:128-40.

9. Horan T.C., Gaynes R.P., Martone W.J., et al. CDC definitions of nosocomial surgical wound infections,1992: a modification of CDC definitions of surgical wound infections. Infect Control Hosp Epidemiol 1992;13:606-8.

10. Levy I., Leibovici L., Drucker M., et al. A prospective study of Gram-negative bacteremia in children. Pediatr Infect Dis J 1996;15:117-22.

11. Nucci M., Colombo A.L., Silveira F., et al. Risk factors for death in patients with candidemia. Infect Control Hosp Epidemiol 1998;19:846-50.
12. Jensen A.G., Wachmann C.H., Poulsen K.B., et al. Risk factors for hospital - acquired Staphylococcus aureus bacteremia. Arch Intern Med 1999;159:1437-44.

13. Raymond J., Aujard Y. Nosocomial infections in pediatric patients: a european, multicenter prospective study. Infect Control Hosp Epidemiol. 2000;21:260-3.

14. Caldeira A.P., França E., Goulart E.M.A. Mortalidade infantil pósneonatal e qualidade da assitência médica. J Pediatr 2001;77:461-8.

15. Yogaraj J.S., Elward A.M., Fraser V.J. Rate, risk factors and outcomes of nosocomial primary bloodstream infections in pediatric intensive care unit patients. Pediatrics 2002;110:481-5.

16. Einloft P.R., Garcia P.C., Piva J.P., et al. A sixteen-year epidemiological profile of a pediatric intensive care unit. Rev Saúde Púb 2002;36:728-33.

17. Turrini R.N.T. Infecção hospitalar e mortalidade. Rev Escola Enfermagem USP 2002;36:177-83.

18. Conterno L.O., Wey S.B., Castelo A. Staphylococcus aureus bacteremia: comparison of two periods and a predictive model of mortality. Braz J Infect Dis 2002;6:288-97.

19. Arantes A., Carvalho E.S., Medeiros E.A.S., et al. Pediatric risk of mortality and hospital infection. Infect Contr Hosp Epidemiol 2004;25:783-5.

20. Felmet K.A., Hall M.W., Clark R.S., et al. Prolonged lymphopenia, lymphoid depletion, and hypoprolactinemia in children with nosocomial sepsis and multiple organ failure. J Immunol 2005; 174 :3765-72.

21. Grohskopf L.A., Huskins W.C., Sinkowitz-Cochran R.L., et al. Use of antimicrobial agents in United States neonatal and pediatric intensive care patients. Pediatr Infect Dis J 2005;24:766-73.

22. Huang C.H., Jang T.N., Liu C.Y., et al. Characteristics of patients with Burkholderia cepacia bacteremia. J Microbiol Immunol Infect 2001;34:215-9.

23. Martha V.F., Garcia P.C., Piva J.P., et al. Comparação entre dois escores de prognóstico (PRISM e PIM) em unidade de terapia intensiva pediátrica. J Pediatr 2005;81:259-64.

24. Wisplinghoff H., Bischoff T., Tallent S.M., et al. Nosocomial bloodstream infections in US hospitals: analysis of 24,179 cases from a prospective nationwide surveillance study. Clin Infect Dis 2004;39:309-17.

25. Kollef M.H., Sherman G., Ward S., Fraser V.J. Inadequate antimicrobial treatment of infections - A risk factor for hospital mortality among critically ill patients. Chest 1999;115:462-74.

26. Ibrahim E.H., Sherman G., Ward S., et al. The influence of inadequate antimicrobial treatment of blood-stream-infections on patient outcomes in the ICU settings. Chest 2000;118:146-55.

27. Auriti C., Ravà L., Di Ciommo V., et al. Short antibiotic prophylaxis for bacterial infections in a neonatal intensive care unit: a randomized controlled trial. J Hosp Infect 2005;59:292-8.

28. Levy I., Shalit I., Askenazi S., et al. Duration and outcome of persistent candidemia in newborn infants. Pediatr Infect Dis J 2006;49:197-201.

29. Gordon A., Isaacs D. Late onset neonatal Gram-negative bacillary infection in Australia and New Zealand: 1992-2002. Pediatr Infect Dis J 2006;25:25-9.

30. Meric M., Willke A., Caglayan C., Toker K. Intensive care unitacquired infections: incidence, risk factors and associated mortality in a Turkish university hospital. Jpn J Infect Dis 2005; 58:297-302.

31. Sader H.S., Gales A.C., Pfaller M.A., et al. Pathogen frequency and resistance patterns in Brazilian hospitals: Summary of results from three years of the SENTRY antimicrobial surveillance program. Braz J Infect Dis 2001;5:200-14.

32. Boletim Eletrônico da Rede de monitoramento de resistência microbiana. http://www.anvisa.gov.br/divulga/newsletter/ rede_rm/index.asp accessed on June 27, 2007. 